\title{
Symbolic and Analytic Techniques for Resource Analysis of Java Bytecode
}

\author{
David Aspinall ${ }^{1}$, Robert Atkey ${ }^{2}$, Kenneth MacKenzie ${ }^{1}$ and Donald Sannella ${ }^{1}$ \\ 1 School of Informatics, The University of Edinburgh, Edinburgh \\ 2 Computer and Information Sciences, University of Strathclyde, Glasgow
}

\begin{abstract}
Recent work in resource analysis has translated the idea of amortised resource analysis to imperative languages using a program logic that allows mixing of assertions about heap shapes, in the tradition of separation logic, and assertions about consumable resources. Separately, polyhedral methods have been used to calculate bounds on numbers of iterations in loop-based programs. We are attempting to combine these ideas to deal with Java programs involving both data structures and loops, focusing on the bytecode level rather than on source code.
\end{abstract}

\section{Introduction}

The ability to move code and other active content smoothly between execution sites is a key element of modern computing platforms. However, it presents huge security challenges, aggravating existing security problems and presenting altogether new ones. One challenging security issue in this context is control of resources (space, time, etc.), particularly on small devices, where computational power and memory are very limited.

A promising approach to security is proof-carrying code [31], whereby mobile code is equipped with an independently verifiable certificate consisting of a condensed proof of its security properties. A major advantage of this approach is that it sidesteps the difficult issue of trust: there is no need to trust either the code producer, or a centralized certification authority. Work on the PCC approach to resource security includes [35] and [7].

This approach requires infrastructure on the side of the code producer as well as the code consumer. The code producer needs to produce not just downloadable code, as before, but also a proof of its security properties. The code consumer needs a way of checking such proofs. Arbitrarily complex methods may be used by the code producer to construct proofs, while their verification by the code consumer is a straightforward check of validity. The burden for the code producer is considerably eased by the use of a certifying compiler which employs static analysis of the source code alongside standard compilation to supply the information required to produce these proofs automatically. The information provided by the analysis - in the case of resource analysis, concerning upper bounds on usage of space, time, etc. - is potentially of great interest to the code producer as an aid to the development of high-quality code, prior to and independent of its use for producing security certificates. 
Recent developments in static analysis methods now makes it feasible to consider an alternative but related approach to security. Instead of requiring the code producer to supply a proof, whether via static analysis of source code or by other means, one can perform an analogous analysis directly on the downloadable bytecode to determine its properties. This could be done by the code consumer on receipt of downloadable code, dispensing with the need for a proof. Alternatively, the code producer could perform the analysis and use the result to produce a proof certificate. An interesting third alternative is that an intermediary, for example a software distributor, could perform such an analysis on uncertified bytecode, transforming it to proof-carrying code. The fact that the original source code is not required is essential to making this feasible in commercial practice.

Here we consider two quite different approaches to the analysis of resource consumption of Java bytecode. The first, in $\S 2$, translates the idea of amortised resource analysis to imperative languages to enable automated resource analysis of programs that iterate through data structures. The second, in $\S 3$, uses polyhedral methods to calculate resource bounds of iterative procedures controlled by numerical quantities. In $\S 4$ we briefly describe some ideas for future work and plans for integrating the two kinds of analysis to deal with Java programs involving both data structures and loops.

\section{Amortised Resource Analysis}

Amortised resource analysis is a technique for specifying and verifying resource bounds of programs by exploiting the tight link between the structure of the data that programs manipulate and the resources they consume. For instance, a program that iterates through a list doing something for every element can either be thought of as requiring $n$ resources, where $n$ is the length of list, or as requiring 1 resource for every element of the list, where we never know the global length property of the list. Taking the latter view can simplify both the specification and the verification of programs' resource usage.

This work conceptually builds on the work of Tarjan and Sleator on amortised complexity analysis [36], where "credits" and "debits" may be virtually

stored within data structures and used to pay for expensive operations. By storing up credit for future operations in a data structure, we amortise the cost of operations on the data structure over time. Hofmann and Jost [21] applied this technique to first-order functional programs to yield an automated resource analysis. Atkey [3] has recently adapted this work to integrate with Separation Logic $[22,34]$ to extend the automated technique to pointer-manipulating imperative programs. In this section we give an overview of Atkey's work and describe some examples.

\subsection{Integrating the Banker's Method and Separation Logic}

Separation Logic is built upon a notion of resources and their separation. The assertion $A * B$ holds for a resource if it can be split into two resources that 
make $A$ true and $B$ true respectively. Resource separation enables local reasoning about mutation of resources; if the program mutates the resource associated with $A$, then we know that $B$ is still true on its separate resource.

For the purposes of complexity analysis, we want to consider resource consumption as well as resource mutation, e.g. the consumption of time as a program executes. To see how Separation Logic-style reasoning about resources helps in this case, consider the standard inductively defined list predicate from Separation Logic, augmented with an additional proposition $R$ denoting the presence of a consumable resource for every element of the list:

$$
\begin{aligned}
\operatorname{list}_{\mathrm{R}}(x) \equiv & x=\text { null } \wedge \text { emp } \\
& \vee \exists y, z \cdot[x \stackrel{\text { data }}{\mapsto} y] *[x \stackrel{\text { next }}{\mapsto} z] * R * \operatorname{list}_{\mathrm{R}}(z)
\end{aligned}
$$

See Atkey [3] for a complete description of the assertion logic. We can represent a heap $H$ and a consumable resource $r$ that satisfy this predicate graphically:

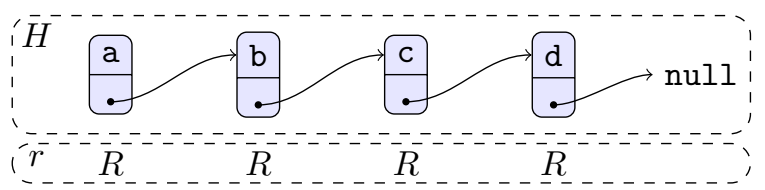

So we have $r, H \models \operatorname{list}_{\mathrm{R}}(x)$, assuming $x$ points to the head of the list. Here $r=R \cdot R \cdot R \cdot R$-we assume that consumable resources form a commutative monoid - and $r$ represents the resource that is available for the program to use in the future. We can split $H$ and $r$ to separate out the head of the list with its associated resource:

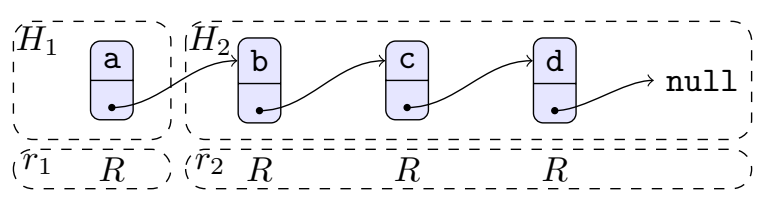

This heap and resource satisfy $r_{1} \cdot r_{2}, H_{1} \uplus H_{2}=[x \stackrel{\text { data }}{\longmapsto} \mathrm{a}] *[x \stackrel{\text { next }}{\longmapsto} y] * R * \operatorname{list}_{\mathrm{R}}(y)$, where $H_{1} \uplus H_{2}=H, r_{1} \cdot r_{2}=r$ and we assume that $y$ points to the $\mathrm{b}$ element. Now that we have separated out the head of the list and its associated consumable resource, we are free to mutate the heap $H_{1}$ and consume the resource $r_{1}$ without affecting the tail of the list, so the program can move to a state:

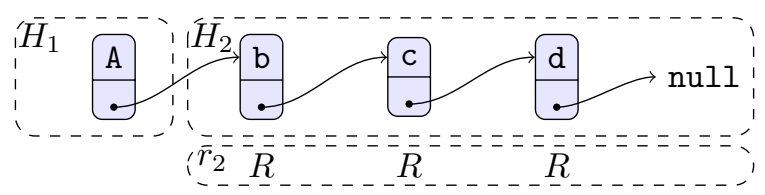

where the head of the list has been mutated to A and the associated resource has been consumed; we do not need to do anything special to reason that the tail of the list and its associated consumable resource are unaffected. 
The combined assertion about heap and consumable resource describes the current shape and contents of the heap and also the available resource that the program may consume in the future. By ensuring that, for every state in the program's execution, the resource consumed plus the resource available for consumption in the future is less than or equal to a predefined bound, we can ensure that the entire execution is resource bounded.

Intermixing resource assertions with Separation Logic assertions about the shapes of data structures, as we have done with the resource-carrying list $\mathrm{R}_{\mathrm{R}}$ predicate above, allow us to specify amounts of resource that depend on the shape of data structures in memory. By the definition of list $t_{R}$, we know that the amount of resource available to the program is proportional to the length of the list, without having to do any arithmetic reasoning about lengths of lists. The association of resources with parts of a data structure is exactly the banker's approach to amortised complexity analysis proposed by Tarjan [36].

In the exposition above we have used a list predicate $\operatorname{list}_{\mathrm{R}}(x)$ that describes a list on the heap with a fixed number of resources per element. Using this predicate only allows the specification of resource usage that is linear in the lengths of lists. Recent work by Hoffmann and Hofmann [20] on amortised resource analysis for polynomial bounds lifts this restriction. Preliminary experiments with combining the two techniques have been promising.

\subsection{Implementation}

The combination of Separation Logic and amortised resource analysis has been implemented in two stages. We have formalised and mechanically checked a proof of soundness for the combined program logic for a simplified subset of Java bytecode in Coq with a shallowly embedded assertion logic. On top of this we have implemented a Coq-verified verification condition generator for a deeply embedded assertion logic and extracted this to OCaml. In OCaml we have implemented a proof search procedure that solves verification conditions using a similar technique to other automated verification tools for Separation Logic [11]. See Atkey [3] for more details. In our proof search implementation, we can leave resource annotations, e.g. the resource associated with each element of a list, as variables to be filled in by a linear program solver. Our tool requires annotation of programs with loop invariants, but can infer the resource portion. This process is demonstrated in the next section.

\subsection{A More Complex Example}

The example shown in the previous section, where a program iterates through a list consuming resources as it proceeds, only demonstrates an extremely simple, albeit common, pattern. We now describe a more complex list manipulating program that shows the benefits of the amortised approach. This example demonstrates the combination of reasonably complex pointer manipulation with resource reasoning. Most of the technical details arise from dealing with the 
heap-shape behaviour of the program; the resource bounds simply drop out of shape constraints thanks to the inference of resource annotations.

Consider the Java method declaration shown in Figure $1^{3}$ that describes the inner loop of an in-place merge sort algorithm for linked lists. The method takes two arguments: list, a reference to the head node of a linked list; and $\mathrm{k}$, an integer. The integer argument dictates the sizes of the sublists that the method will be merging in this pass. In short, the method steps through the list $2 * \mathrm{k}$ elements at a time, merging the two length $\mathrm{k}$ sublists each time. The outer loop does the $2 * \mathrm{k}$ stepping, and the inner loop does the merging. To accomplish a full merge sort, this method would be called $\log _{2}(n)$ times with doubling $\mathrm{k}$, where $n$ is the length of the list.

Assume that we wish to account for the number of swapping operations performed by this method, i.e. the number of times that the third branch of the if statement in the inner loop is executed. We accomplish this in our implementation by inserting a special consume instruction at this point.

The pre- and post-conditions of the method are as follows:

$$
\begin{aligned}
& \text { Pre }(\text { mergeInner }): \text { list } \neq \text { null } \wedge\left(\operatorname{lseg}(x, \text { list }, \text { null }) * R^{y}\right) \\
& \text { Post(mergeInner }): l \operatorname{seg}(0, \text { retval }, \text { null })
\end{aligned}
$$

The precondition states that the first argument points to a list segment ending with null, with $x$ amount of resource associated with every element of the list, and $y$ amount of additional resource that may be used. The values of $x$ and $y$ will be inferred by a linear program solver. The condition list $\neq$ null is a safety condition required for the method to not throw a null pointer exception.

The outer loop in the method needs a disjunctive invariant corresponding to whether this is the first iteration or a later iteration.

$$
\begin{aligned}
& \left(\operatorname{lseg}\left(o_{1}, \text { list }, \text { tail }\right) *[\text { tail } \stackrel{\text { next }}{\longmapsto} ?] *[\operatorname{tail} \stackrel{\text { data }}{\mapsto} ?] * \operatorname{lseg}\left(o_{2}, \mathrm{p}, \operatorname{null} l\right) * R^{o_{3}}\right) \\
& \vee\left((\text { list }=\operatorname{null} \wedge \text { tail }=\operatorname{null}) * l \operatorname{seg}\left(o_{4}, \mathrm{p}, \operatorname{null}\right) * R^{o_{5}}\right)
\end{aligned}
$$

The first disjunct is used on normal iterations of loop: the variable list points to the list that has been processed so far, ending at tail; p points to the remainder of the list that is to be processed. We have annotated these lists with the resource variables $o_{1}$ and $o_{2}$ that will contain the resources associated with each element of these lists. The second disjunct covers the case of the first iteration, when list and tail are null and p points to the complete list to be processed.

Moving on, we consider the first inner loop that advances the pointer $\mathrm{q}$ by $\mathrm{k}$ elements forward, thus splitting the list ahead of $\mathrm{p}$ into a $\mathrm{k}$-element segment and the rest of the list. The next loop will merge the first k-length segment with the $\mathrm{k}$-length prefix of the second segment. It is convenient for our implementation to split out this inner loop into another method ${ }^{4}$, with the following signature:

3 Adapted from the $\mathrm{C}$ code at http://www. chiark. greenend.org.uk/ sgtatham/algorithms/listsort.html.

4 This is because our implementation works on unstructured bytecode, and so cannot easily apply Separation Logic's frame rule to modularise the reasoning about the loop. Using a separate method allows application of the frame rule. 


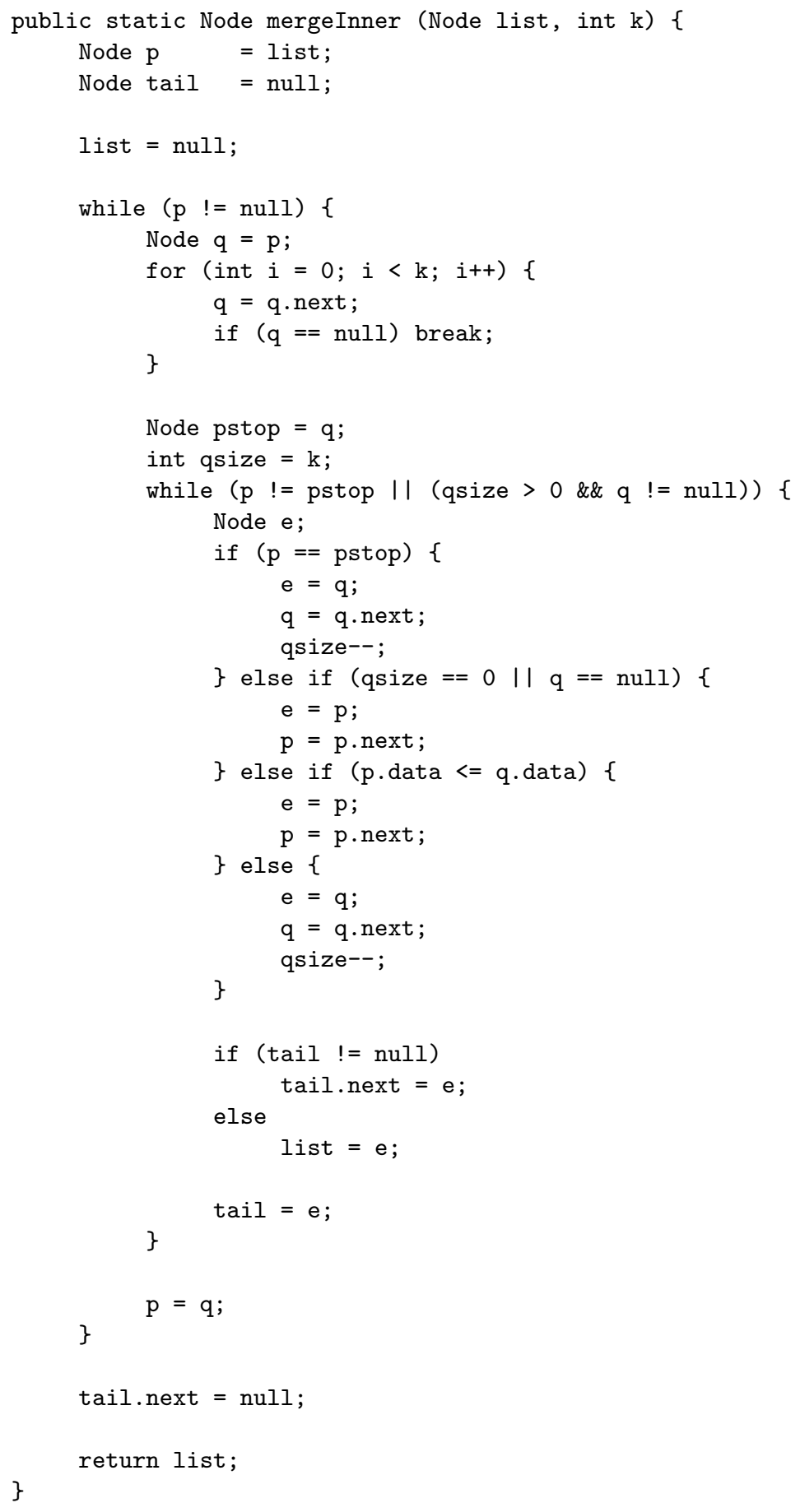

Fig. 1. Inner loop of an in-place linked-list merge sort 
public static Node advance (Node 1 , int $\mathrm{k}$ )

The argument 1 points to a linked list, and the method will advance $\mathrm{k}$ elements through the list (or until the end) and return a pointer to the split point. The pre- and post-condition of this method are:

$$
\begin{aligned}
\text { Pre }(\text { advance }) & : \operatorname{lseg}\left(a_{0}, 1, \text { null }\right) \\
\text { Post (advance }) & : \operatorname{lseg}\left(a_{0}, 1, \text { retval }\right) * l \operatorname{seg}\left(a_{0}, \text { retval }, \text { null }\right)
\end{aligned}
$$

Again, we have left the resource annotation on the elements of the list as a variable $a_{0}$, to be filled in by the linear solver. The appearance of the same variable in the pre- and post-condition implies that we expect this resource to be preserved by the method.

Proceeding though our main method, the invariant of the inner loop is as follows, again in two pieces according to whether it is the first or second iteration of the outer loop:

$$
\begin{gathered}
\left(\operatorname{lseg}\left(i_{1}, \text { list, tail }\right) *[\operatorname{tail} \stackrel{\text { next }}{\mapsto} ?] *[\operatorname{tail} \stackrel{\text { data }}{\mapsto} ?]\right. \\
\left.* \operatorname{lseg}\left(i_{2}, \mathrm{p}, \operatorname{pstop}\right) * \operatorname{lseg}\left(i_{3}, \mathrm{q}, \operatorname{null}\right) * R^{i_{4}}\right) \\
\vee\left((\text { list }=\operatorname{null} \wedge \text { tail }=\operatorname{null}) * \operatorname{lseg}\left(i_{5}, \mathrm{p}, \operatorname{pstop}\right) * \operatorname{lseg}\left(i_{6}, \mathrm{q}, \operatorname{null}\right) * R^{i_{7}}\right)
\end{gathered}
$$

The first part of each disjunct is as before, stating that list to tail contains the part of list that has been processed. Since we have now split the remainder of the list into two pieces we have two separate list segments referenced by $\mathrm{p}$ and q pointing to the parts of the list that are to be merged.

Running this example through our implementation produces the solution $x=1, y=0$ for the precondition resource annotations. This indicates that each element of the list needs to contain one resource for every element. For the outer loop's invariant, we obtain $o_{2}=o_{4}=1$ and all the others are 0 . This indicates that the list we have processed has had all its resources consumed, while the list remaining to be processed still has associated resources. This is as expected for a loop iterating through a list. The specification of advance is completed by inferring $a_{0}=1$, indicating that advance preserves the resources associated with the list. Finally the inner loop's invariant has $i_{2}=i_{3}=i_{5}=i_{6}=1$ and all others 0 , indicating that the two list segments that are remaining to be processed have associated resources, while the processed segments do not.

Comparisons to other techniques. While we have had to work to supply the loop invariants for our implementation, we note that these invariants may be inferred by other tools, for example [11], and the resource variables automatically inserted on the list segment parts. The key to the amortised approach is the tight connection between shape invariants, which is a complex but well-studied problem, and resource usage.

Most other techniques for resource usage analysis that handle data structures do so by considering the sizes of structures. The SPEED system of Gulwani et al [19] can infer resource bounds for programs manipulating heap-based data 
structures, but only via abstract interfaces. The specifications for these abstract interfaces record the effect of the operations on the size of the data structure. Thus, the technique is unable to cope with the kind of program that we have presented above that uses direct pointer manipulation. Nevertheless, Gulwani et al report impressive results on real-world Microsoft product code.

The COSTA system [2] can deal with some uses of direct pointer manipulation, but accounts for the sizes of heap-based data structures by counting the length of the longest path from a given reference. Thus, it cannot deal with programs that demonstrate sharing on the heap; the Java method described above has three pointers all pointing the same list in the inner loop.

One might also consider the use of Separation Logic to deal with sharing on the heap, augmented with information on the sizes of heap-base data structures to account for resource usage. So one would have a predicate $l s e g^{n}(x, y)$ that describes a list segment of length $n$ from $x$ to $y$, plus a "ghost variable" that accounts for the consumed resources. We argue that the amortised approach described here is simpler due to the differences in reasoning between the global property of the length of a whole list, and the local property of each list element having an associated amount of resource to be used. For example, consider the specification of the advance method using sized structures:

$$
\begin{aligned}
& \text { Pre }(\text { advance }): \operatorname{lseg}^{n}(1, \text { null }) \\
& \text { Post }(\text { advance }): \exists n_{1}, n_{2} \cdot n_{1}+n_{2}=n \wedge\left(\operatorname{lseg}^{n_{1}}(1, \text { retval }) * l \operatorname{seg}^{n_{2}}(\text { retval, null })\right)
\end{aligned}
$$

We have had to introduce two existential variables indicating the sizes of the lists returned by the method. These additional values have to then be related back to the length of the original list by the calling method, and thence to the resource consumption, requiring non-straightforward arithmetic reasoning. The amortised approach exploits the shape-reasoning already present in Separation Logic to account for resources. For further elaboration of this point, and a demonstration of the use of amortised specification to improve information hiding in specifications, see the functional queues example in [3].

\section{$3 \quad$ Iteration and geometry}

The previous section has described a technique which can be used to analyse the resource usage of procedures which manipulate heap-based data structures. Here we will describe a mathematical technique which can be used to study iterative procedures controlled by numerical quantities. One of our main interests is in producing certifying analyses, and our description of the mathematics will highlight aspects which are relevant to this problem.

We will look at some examples of Java methods which use iteration. For simplicity, we will look at the problem of deciding how often the println method is called, but we could equally be looking at object allocation or the transmission of SMS messages.

Here is an example with nested loops: 


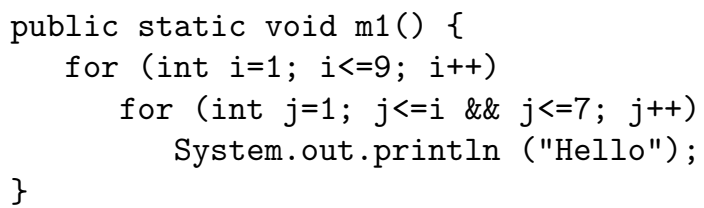

For a more complicated example, consider this Java method where both loops are controlled by method arguments:

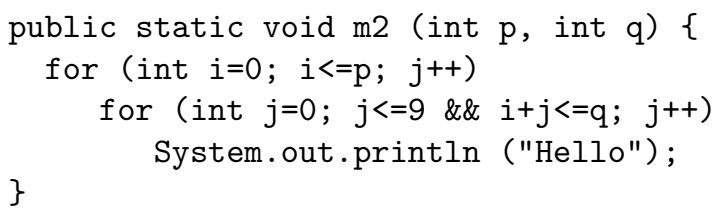

How can one tell how many times println is called in these methods? Consider m1 again. Every time we visit the println statement we have the following constraints on the program variables $i$ and $j$ :

$$
\begin{gathered}
1 \leq i \leq 9 \\
1 \leq j \leq i \\
1 \leq j \leq 7 .
\end{gathered}
$$

Considered as inequalities over the real numbers, these define a trapezoidal region $P$ in the $(i, j)$-plane, and it is easy to see that the number of times the println statement is executed is equal to $\left|P \cap \mathbb{Z}^{2}\right|$, the number of lattice points ${ }^{5}$ within the polygon $P$.

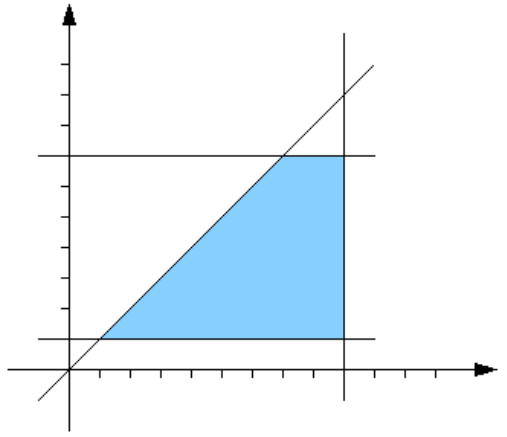

Fig. 2. Polygon $P$ for method $\mathrm{m} 1$

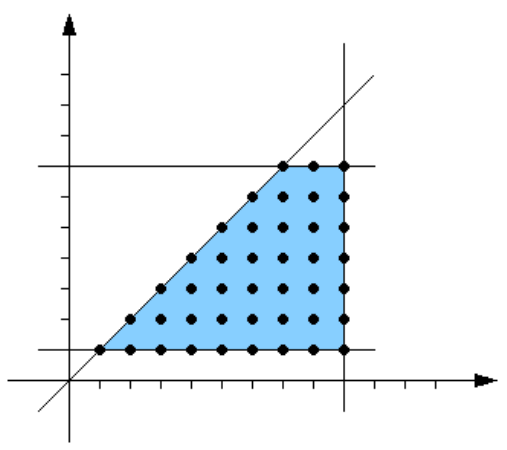

Fig. 3. Lattice points in $P$

There is a rich mathematical theory of the enumeration of lattice points in polytopes (the generalisation of polygons to higher dimensions) and we will describe some aspects of this theory and its relations to program analysis.

\footnotetext{
${ }^{5}$ i.e. points with integral coordinates.
} 


\subsection{Halfspaces, polyhedra, and polytopes}

Fix an integer $d \geq 0$ and $a_{1}, \ldots, a_{d} \in \mathbb{R}$. We will be interested in solutions $\left(x_{1}, \ldots, x_{n}\right) \in \mathbb{R}^{d}$ of inequalities of the form

$$
a_{1} x_{1}+\cdots+a_{d} x_{d} \leq b .
$$

In our applications, such inequalities will arise in the form of linear constraints on program variables. Putting $\mathbf{a}=\left(a_{1}, \ldots, a_{d}\right)$ and $\mathbf{x}=\left(x_{1}, \ldots, x_{d}\right)$ we can rewrite (1) as $\mathbf{a} \cdot \mathbf{x} \leq b$, and if $\mathbf{a} \neq \mathbf{0}$ then the set of $\mathbf{x}$ satisfying the inequality defines a halfspace in $\mathbb{R}^{d}$. For example, in $\mathbb{R}^{2}$ a halfspace consists of all points lying on one side of some line.

A convex polyhedron in $\mathbb{R}^{d}$ is the intersection of a finite number of halfspaces, and a bounded polyhedron (a polyhedron of finite extent, i.e. one which is contained in some sphere) is called a polytope. It can be shown that a polytope can equivalently be defined as the convex hull ${ }^{6}$ of a finite set of points in $\mathbb{R}^{d}$ (the vertices of $P$ ). Moreover, if the constants in the inequalities defining $P$ are all rational (as will be the case in all of our applications), the vertices of $P$ all have rational co-ordinates. A convex polyhedron is thus the set of simultaneous solutions to a system of $n$ inequalities:

$$
\begin{aligned}
a_{11} x_{1}+\cdots+a_{1 d} x_{d} & \leq b_{1} \\
a_{21} x_{1}+\cdots+a_{2 d} x_{d} & \leq b_{2} \\
\vdots & \\
a_{n 1} x_{1}+\cdots+a_{n d} x_{d} & \leq b_{n} .
\end{aligned}
$$

The general theory of polyhedra has many applications in mathematics and in computer science. See [6] for a survey of applications in computer science.

Note that if we restrict to natural numbers, then linear inequalities of the type considered above are exactly the type of inequalities that occur in Presburger arithmetic. It follows that the lattice point enumeration problem subsumes the problem of counting solutions to systems of Presburger inequalities. This point of view is examined in greater depth by Pugh in [33].

\subsection{Ehrhart Polynomials}

Many applications of polytope methods have been based on the work of Eugène Ehrhart $[17,18]$, who studied the problem of how the number of lattice points inside a polytope grows as the size of the polytope increases. More precisely, let

$$
P=\operatorname{conv}\left\{\mathbf{y}_{1}, \ldots, \mathbf{y}_{m}\right\}
$$

be a polytope and for $n \in \mathbb{N}$, let

$$
n P=\operatorname{conv}\left\{n \mathbf{y}_{1}, \ldots, n \mathbf{y}_{m}\right\}
$$

\footnotetext{
${ }^{6}$ We denote the convex hull of a set $X$ by conv $X$
} 
be the $n$-fold dilate of $P$. Ehrhart showed that $\left|n P \cap \mathbb{Z}^{d}\right|$ is a quasipolynomial in $n$, which may be thought of as a number of polynomials cyclically interleaved.

Definition. A quasipolynomial of degree $d$ is a function $f: \mathbb{Z} \rightarrow \mathbb{Z}$ of the form

$$
f(n)= \begin{cases}f_{0}(n) & \text { if } n \equiv 0 \quad(\bmod k) \\ f_{1}(n) & \text { if } n \equiv 1 \quad(\bmod k) \\ & \vdots \\ f_{k-1}(n) & \text { if } n \equiv k-1 \quad(\bmod k) .\end{cases}
$$

where each $f_{j}$ is a polynomial of the usual kind and $\max \left\{\operatorname{deg} f_{0}, \ldots, \operatorname{deg} f_{k-1}\right\}=$ $d$. The (minimal) number $k$ of polynomial components is called the quasiperiod of $f$.

Theorem. Let $P=\operatorname{conv}\left\{\mathbf{y}_{1}, \ldots, \mathbf{y}_{n}\right\}$ be a rational convex polytope in $\mathbb{Z}^{d}$ and let

$$
\mathcal{E}_{P}(n)=\left|n P \cap \mathbb{Z}^{d}\right| .
$$

Then $\mathcal{E}_{P}(n)$ is a quasipolynomial of degree $\operatorname{dim} P$ and quasiperiod equal to the greatest common denominator of the coordinates of the vertices of $P$.

The original proof of this theorem can be found in [17]; see also [9, Chapter 3].

There is a considerable amount of research applying Ehrhart polynomials to program analysis and optimisation, especially in the field of high-performance computing involving array calculations. One of the first papers in this area is due to Clauss [14], with application to problems such as counting the flops executed by a loop, the number of memory locations touched by a loop, the array elements that must be transmitted from one processor to another during parallel array computations, the maximum parallelism induced by a loop from a given timeschedule, and several others. Further work appears in $[25,15,38]$ for example.

The methods of Clauss seem to have remained largely within the highperformance/parallel computing community (see [24,32] for example) until 2006, when Braberman et al [13] (and see also [12]) showed how to adapt these techniques to predict the memory usage of (iterative) Java programs; at present this appears to be the only application of polytope methods within the programming language community.

\subsection{Drawbacks of Ehrhart polynomials}

The standard method used to compute Ehrhart polynomials is interpolation, where the coefficients of a polynomial $f$ of degree $d$ are derived from the values of the polynomial at $d+1$ distinct points: this data gives a $(d+1) \times(d+1)$ system of linear equations in the coefficients of $f$ which can then be solved by Gaussian elimination or some other technique. In the case of a quasipolynomial of period $k$ and degree $d$, this requires us to solve $k$ systems of $(d+1) \times(d+1)$ equations. Recalling that the period $k$ of the Ehrhart polynomial associated with a rational 
polytope $P$ is the greatest common denominator of the coefficients of the vertices of $P$, it becomes clear that a considerable amount of computation can be required to calculate $\mathcal{E}_{P}(n)$. In addition to this, the initial $d+1$ values of the $k$ polynomial components of the quasipolynomial have to be computed by explicitly counting the number of lattice points in the dilates $0 P, P, 2 P, \ldots,(d+1) P$. The number $k$ can be very large, even for relatively simple polytopes. For example, for the triangular polytope

$$
P=\operatorname{conv}\left\{\left(\frac{1}{4}, \frac{2}{5}\right),\left(\frac{5}{7}, \frac{2}{11}\right),\left(\frac{8}{9}, \frac{1}{12}\right)\right\}
$$

the quasiperiod of $\mathcal{E}_{P}(n)$ is 13,680 . Calculating the Ehrhart polynomial of $P$ thus requires the solution of $13,6803 \times 3$ systems of linear equations, which would be reasonably time-consuming. In fact, even if the dimension $d$ is fixed, the time taken to compute (via interpolation) the Ehrhart polynomial of a polytope with $n$ vertices can grow exponentially with $n$ (see $[38, \S 2.3]$ ), whereas the methods presented in the next section are polynomial in fixed dimension.

The sheer amount of data required to specify an Ehrhart function is also something of a barrier in the context of certified resource analysis, where such functions would have to be recorded in certificates accompanying mobile programs. This may not in fact be an insurmountable problem. One could possibly find simpler functions which are upper bounds for the exact Ehrhart function (see [30]); this would save space at the expense of a (hopefully small) loss of precision. Another issue is that Ehrhart functions are not arbitrary quasipolynomials: for example it is clear that they are increasing functions, whereas a general quasipolynomial can have polynomial components which are completely unrelated, leading to a function whose value oscillates drastically. It is conceivable that the quasipolynomials arising as Ehrhart functions have special properties which would enable them to be specified by a relatively small amount of data. Unfortunately, it seems that very little is known about exactly which quasipolynomials can occur as Ehrhart polynomials (see $[28,10]$ for some partial results) so at present it is difficult to be precise about the minimum of data required to explicitly specify an Ehrhart function. However, the results discussed in the next section may enable us to bypass this problem.

\subsection{Generating functions}

The difficulty of computing Ehrhart polynomials suggests that they would be unsuitable for polytope-based analyses in a certifying framework, but fortunately some more recent results provide a much more efficient means of enumerating lattice points. The basic tool in this theory is the generating function of a polytope, which is a multivariate polynomial with a term for every lattice point in the polytope. More concretely, suppose we have a polytope $P$ in $R^{d}$. We will consider polynomials in the variables $x_{1}, \ldots, x_{d}$. Given $\mathbf{v}=\left(v_{1}, \ldots, v_{d}\right) \in \mathbb{Z}^{d}$ we define

$$
\mathbf{x}^{\mathbf{v}}=x_{1}^{v_{1}} x_{2}^{v_{2}} \cdots x_{d}^{v_{d}}
$$


and the generating function of $P$ is then defined by

$$
\mathcal{G}_{P}(\mathbf{x})=\sum\left\{\mathbf{x}^{\mathbf{v}}: \mathbf{v} \in P \cap \mathbb{Z}^{d}\right\}
$$

It is easy to see that the number of lattice points in $P$ is given by $\mathcal{G}_{P}(1, \ldots, 1)$. The obvious difficulty here is that the polynomial $\mathcal{G}_{P}(\mathbf{x})$ will in general be enormous and costly to compute. Recall our earlier example, which gave rise to a trapezoidal region in $\mathbb{R}^{2}$ :

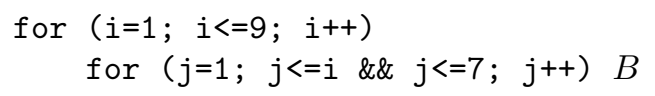

For this relatively small example, the full generating function is

$$
\begin{array}{r}
\mathcal{G}_{P}(x, y)=x y+x^{2} y+x^{3} y+x^{4} y+x^{5} y+x^{6} y+x^{7} y+x^{8} y+x^{9} y \\
+x^{2} y^{2}+x^{3} y^{2}+x^{4} y^{2}+x^{5} y^{2}+x^{6} y^{2}+x^{7} y^{2}+x^{8} y^{2}+x^{9} y^{2} \\
+x^{3} y^{3}+x^{4} y^{3}+x^{5} y^{3}+x^{6} y^{3}+x^{7} y^{3}+x^{8} y^{3}+x^{9} y^{3} \\
+x^{4} y^{4}+x^{5} y^{4}+x^{6} y^{4}+x^{7} y^{4}+x^{8} y^{4}+x^{9} y^{4} \\
+x^{5} y^{5}+x^{6} y^{5}+x^{7} y^{5}+x^{8} y^{5}+x^{9} y^{5} \\
+x^{6} y^{6}+x^{7} y^{6}+x^{8} y^{6}+x^{9} y^{6} \\
+x^{7} y^{7}+x^{8} y^{7}+x^{9} y^{7}
\end{array}
$$

which is already quite unwieldy.

However, Alexander Barvinok [8] has recently shown how to express the generating function as a sum of short rational functions which are easily determined from local information at the vertices of $P$. In the case above, we have

$$
\begin{aligned}
\mathcal{G}_{P}(x, y) & =\frac{x y}{(1-x)(1-x y)}+\frac{x^{9} y}{\left(1-x^{-1}\right)(1-y)} \\
& +\frac{x^{9} y^{7}}{\left(1-y^{-1}\right)\left(1-x^{-1}\right)}+\frac{x^{7} y^{7}}{(1-x)\left(1-x^{-1} y^{-1}\right)}
\end{aligned}
$$

This function is easily computed if one knows the vertices and edges of the polytope. Space constraints prevent us from describing the computation in detail here, but a full explanation can be found in [8] or [9].

There is a problem here, though. To find $\left|P \cap \mathbb{Z}^{2}\right|$ we have to evaluate $\mathcal{G}_{P}(1,1)$, and the denominators of all of the terms above vanish at $(1,1)$. However, this can be overcome. The singularity at $(1,1)$ is a removable singularity $[1, \S 3.1]$, and various techniques can be used to find $\lim _{(x, y) \rightarrow(1,1)} \mathcal{G}_{P}(x, y)$. For example, we can find a common denominator to obtain

$$
\begin{aligned}
\mathcal{G}_{P}(x, y) & =\frac{x y-x y^{2}-x^{10} y+x^{11} y^{2}+x^{10} y^{8}-x^{11} y^{9}-x^{8} y^{8}+x^{8} y^{9}}{(1-x)(1-y)(1-x y)} \\
& =\frac{x y-x y^{2}-x^{10} y+x^{11} y^{2}+x^{10} y^{8}-x^{11} y^{9}-x^{8} y^{8}+x^{8} y^{9}}{1-x-y+x^{2} y+x y^{2}-x^{2} y^{2}}
\end{aligned}
$$


and then repeatedly apply L'Hôpital's rule ${ }^{7}$ to obtain

$$
\begin{aligned}
\left|P \cap \mathbb{Z}^{2}\right| & =\mathcal{G}_{P}(1,1) \\
& =\lim _{(x, y) \rightarrow(1,1)} \frac{x y-x y^{2}-x^{10} y+x^{11} y^{2}+x^{10} y^{8}-x^{11} y^{9}-x^{8} y^{8}+x^{8} y^{9}}{1-x-y+x^{2} y+x y^{2}-x^{2} y^{2}} \\
& =\lim _{(x, y) \rightarrow(1,1)} \frac{\frac{\partial}{\partial y}\left(x y-x y^{2}-x^{10} y+x^{11} y^{2}+x^{10} y^{8}-x^{11} y^{9}-x^{8} y^{8}+x^{8} y^{9}\right)}{\frac{\partial}{\partial y}\left(1-x-y+x^{2} y+x y^{2}-x^{2} y^{2}\right)} \\
& =\cdots \\
& =\frac{-2+22+560-792-448+576}{-2} \\
& =42
\end{aligned}
$$

which is indeed equal to the number of lattice points in Figure 3.

This calculation may appear to be quite complex in relation to our relatively small example, but it is easy to automate ${ }^{8}$. Note also that the complexity of the calculation depends only on the shape of the polytope, and not its size. If we took a region of a similar shape but many times larger, all that would change would be the exponents of $x$ and $y$ in the numerator of the generating function; the calculation required to determine the number of lattice points would be essentially identical to that above.

We have only considered Barvinok's construction for integral polytopes here, but the theory can be extended to rational polytopes as well. it is also possible to recover most of the theory of Ehrhart polynomials, which is useful for the study of parametric bounds. This approach is developed in detail by De Loera et al in [26], which describes the implementation of Barvinok's techniques in the LattE package. De Loera's work is applied to program analysis problems in [38], where much of Clauss' work is recast in terms of Barvinok's methods. Generating-function methods have recently been applied to the problem of Worst Case Execution Time in [27]. See also [9] for an exposition of the mathematics of the Barvinok theory.

\subsection{Implementation}

We have implemented (in OCaml) a Java compiler which uses lattice point enumeration techniques to calculate resource bounds for simple imperative programs. This is a preliminary implementation, but the results it produces are quite promising; it can successfully (and automatically) produce precise bounds for realistic matrix manipulation programs, for example (see Appendix A for some examples).

$\overline{7}$ If $f$ and $g$ are continuous at $a$ and $\lim _{x \rightarrow a} f(x)=\lim _{x \rightarrow a} g(x)=0$ then $\lim _{x \rightarrow a} f(x) / g(x)=\lim _{x \rightarrow a} f^{\prime}(x) / g^{\prime}(x)$

8 The calculation works particularly well for our example because our polygon is specially shaped; in the general case a more complex (but still tractable) computation is required. 
Inferring linear constraints. The first phase of the compiler converts the source program to an expression-based form in which all names have been resolved. This form is very similar to the source program, and preserves the explicit control-flow structures of Java.

Our first task is to infer systems of linear constraints on program variables. The expression-based form is converted into a control-flow graph and then between every pair of expressions we infer a polyhedron which bounds the values of the integral variables in the program. This is done using a well-known technique due to Cousot and Halbwachs which involves abstract interpretation over a domain of polyhedra. See [16] for details.

A number of polyhedral operations are required to perform this process. It is necessary to have some representation of polyhedra and the means to convert between vertex and facet representations, and methods for combining polyhedra in various ways (intersection, join (polyhedral hull), widening, ... ) are also needed. These can be difficult to program, but fortunately there are a number of high-quality libraries available. We have chosen to use the Parma Polyhedra Library (PPL) [5], which is a large $\mathrm{C}++$ library providing all of the operations we require, including polyhedral widening operators (see [4]) necessary to ensure termination of the abstract interpretation process. The PPL also provides an OCaml interface which was convenient for linking with our OCaml-based compiler.

Using the PPL it was a relatively straightforward task to implement the Cousot-Halbwachs technique and obtain linear bounds on program variables.

Enumerating lattice points. Having determined polytopes controlling loop iteration, it is necessary to enumerate lattice points in order to find bounds on the number of loop executions. We have done this using the barvinok library ${ }^{9}$ of Sven Verdoolaege, which implements the generating-function methods described in $\S 3.4$, and this enables us to automatically find our desired resource bounds.

There are certain difficulties in this approach however; in particular, it can be difficult to decide which variables control iterations, and what the dimension of the relevant polytope should be. Our prototype compiler works with a representation which has a fairly explicit representation of the loop structure of the input program, and we have developed heuristics which enable us to determine the relevant polytopes. This works well in practice, with realistic code examples, but it is possible to devise examples which cause the analysis to give incorrect results. However, we believe that this problem can be solved by methods which will be described below.

\subsection{Analysing compiled bytecode}

We are currently attempting to apply lattice-point methods to the resource analysis of JVM bytecode methods. A basic problem here is that it can be difficult

\footnotetext{
${ }^{9}$ http://freshmeat.net/projects/barvinok/
} 
to determine the precise loop structure of a program by examining the bytecode. Consider the following examples.

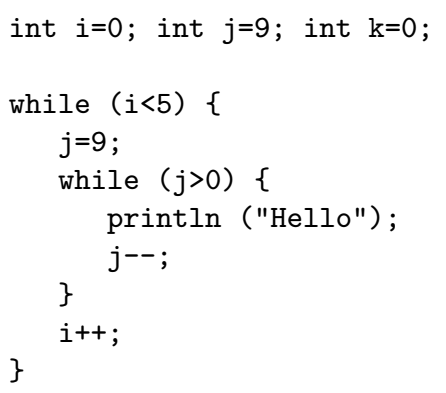

Fig. 4.

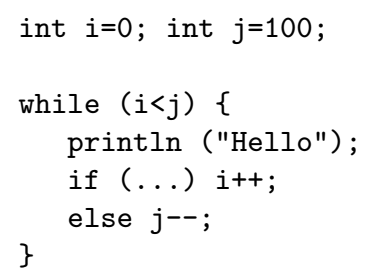

Fig. 5.

In Figure 4, the entire inner loop is executed once for each iteration of the outer loop, and the println method is called a total of 45 times; however, if the statement $\mathrm{j}=9$ is altered to $k=9$ then the "inner" loop is executed once only, so println is executed only 9 times. This example shows that a very small change (only a single instruction in the compiled bytecode will change) can have a major effect on the resource usage of a program. The two versions of the program even have identical control-flow graphs, so it is not easy to see how to perform an accurate analysis of resource usage.

In Figure 5 the loop is controlled by two variables, but the iteration is onedimensional. How can we recognise such patterns?

Instrumenting the code with counters. Gulwani et al [19] have proposed a technique for instrumenting with counter variables which can then be used for resource analysis. The example in Figure 5 would become

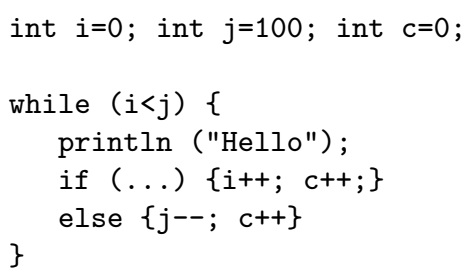

The Cousot-Halbwachs technique can successfully analyse this example to deduce that $0 \leq c \leq 99$, allowing us to conclude that the loop is executed at most 100 times.

An algorithm is described in [19] which automatically discovers a collection of counters which can be used to instrument the back-edges in a control-flow graph and then used to analyse the resource usage. The algorithm also gives 
dependencies between counters which enable one to attack nested structures such as the one in Figure 4 above. However, the results of the analysis can be somewhat imprecise due to the fact that bounds associated with "nested" counters are simply multiplied together to obtain an overall bound.

We believe that the Gulwani algorithm can be refined to provide more precise relations between counters which can then be analysed using lattice-point methods to give more precise bounds on loop iterations.

We are currently implementing an analysis for compiled JVM bytecode which will combine the instrumentation technique of Gulwani with lattice-point methods and amortised analysis, and we hope that this will allow us to automatically analyse the resource consumption of many programs.

\section{Further Work}

The lattice-point techniques described above only apply to single methods. We would like to integrate our work with existing techniques to enable analysis of complete Java applications (including recursion).

Some of the geometrical algorithms are computationally expensive; in particular, the complexity of certain polyhedral operations grows exponentially as the dimension increases. We would like to develop certifying versions of these algorithms so that the output can be verified without excessive effort.

Polyhedral libraries are written in $\mathrm{C}++$ and are very large and complex (PPL is over 100,000 lines long), and also depend on a number of external libraries (for example the gmp library for unlimited-precision arithmetic). This provides a lot of opportunities for bugs to creep in, and certifying algorithms would have the added benefit that they would allow us to be sure of the correctness of the output without having to trust the correctness of the libraries. See [29, 23] for more on this point of view.

One of our motivations is to measure memory consumption of Java programs. A common assumption in research on this topic is that all objects from a given class are of the same size. However, this will not always be the case: for example, the Java BigInteger class represents integers with unlimited precision, and the size of an object will depend on the integer involved. Furthermore, the size of an object returned by a method may depend on the method arguments consider the BigInteger multiply method. We are not aware of any previous research which is able to deal with this type of behaviour. However, there is some recent work by Verdoolaege and Bruynooghe [37] on weighted generating functions for polytopes, in which instead of considering the usual generating function $\sum\left\{\mathbf{x}^{\mathbf{v}}: \mathbf{v} \in P \cap \mathbb{Z}^{d}\right\}$, one considers a function of the form $\sum\left\{f(\mathbf{v}) \mathbf{x}^{\mathbf{v}}\right.$ : $\left.\mathbf{v} \in P \cap \mathbb{Z}^{d}\right\}$ in which each lattice point is weighted according to some function $f$. This corresponds to the situation in which a nest of loops indexed by $i_{1}, \ldots, i_{d}$ allocates an amount of memory given by the function $f\left(i_{1}, \ldots, i_{d}\right)$. It seems

plausible that this work would be useful for attacking the problem of "dependent allocation" of the type discussed above. 
Examination of a large number of examples suggests that most methods which involve loops deal either with iteration over data structures or with iteration controlled by integer variables, but that it is unusual to encounter situations which involve both simultaneously, the most common such situation being the conversion of a list to an array or vice versa. This makes us hopeful that a straightforward combination of our two techniques will enable the automatic analysis of a substantial proportion of Java methods. There are however certain situations where it is difficult to determine the amount of iteration required in advance - for example, worklist algorithms where processing one element of a queue may add an unpredictable number of new elements to the end of the queue, or iterative floating-point numerical algorithms where the number of iterations required is very sensitive to input data - and these remain beyond the scope of our methods at present.

\section{Acknowledgments}

This work was funded in part by the Sixth Framework programme of the European Community under the MOBIUS project FP6-015905. This report reflects only the author's views and the European Community is not liable for any use that may be made of the information contained therein.

This work was funded in part by the ReQueST grant (EP/C537068) from the EPSRC e-Science Programme, and by the RESA grant (EP/G006032/1) from the EPSRC Follow-on Fund.

\section{References}

1. Lars Ahlfors. Complex Analysis. International Series in Pure and Applied Mathematics. McGraw-Hill, 1979.

2. Elvira Albert, Puri Arenas, Samir Genaim, German Puebla, and Damiano Zanardini. COSTA: Design and implementation of a cost and termination analyzer for Java bytecode. In Formal Methods for Components and Objects, 6th International Symposium, FMCO 2007, Revised Lectures, volume 5382 of Lecture Notes in Computer Science, pages 113-132. Springer, 2007.

3. Robert Atkey. Amortised resource analysis with separation logic. In ESOP 2010: Proceedings of the 19th European Symposium on Programming Languages and Systems, volume 6012 of Lecture Notes in Computer Science, pages 85-103. Springer, 2010.

4. R. Bagnara, P. M. Hill, E. Ricci, and E. Zaffanella. Precise widening operators for convex polyhedra. Science of Computer Programming, 58(1-2):28-56, 2005.

5. R. Bagnara, P. M. Hill, and E. Zaffanella. The Parma Polyhedra Library: Toward a complete set of numerical abstractions for the analysis and verification of hardware and software systems. Science of Computer Programming, 72(1-2):3-21, 2008.

6. Roberto Bagnara, Patricia M. Hill, and Enea Zaffanella. Applications of polyhedral computations to the analysis and verification of hardware and software systems. Theor. Comput. Sci., 410(46):4672-4691, 2009. 
7. Gilles Barthe, Lennart Beringer, Pierre Crégut, Benjamin Grégoire, Martin Hofmann, Peter Müller, Erik Poll, Germán Puebla, Ian Stark, and Eric Vétillard. MOBIUS: Mobility, ubiquity, security — objectives and progress report. In Trustworthy Global Computing: Revised Selected Papers from the Second Symposium TGC 2006, number 4661 in Lecture Notes in Computer Science. Springer-Verlag, 2007.

8. Alexander Barvinok and James E. Pommersheim. An algorithmic theory of lattice points in polyhedra. In New Perspectives in Algebraic Combinatorics (Berkeley, CA, 1996-97), volume 38 of Math. Sci. Res. Inst. Publ., pages 91-147. Cambridge Univ. Press, 1999.

9. Matthias Beck and Sinai Robins. Computing the Continuous Discretely. Undergraduate Texts in Mathematics. Springer-Verlag, 2007.

10. Matthias Beck, Steven Sam, and Kevin Woods. Maximal periods of (Ehrhart) quasi-polynomials. J. Combin. Theory Ser. A, 115:517-525, 2008.

11. Josh Berdine, Cristiano Calcagno, and Peter W. O'Hearn. Symbolic execution with separation logic. In Kwangkeun Yi, editor, APLAS, volume 3780 of Lecture Notes in Computer Science, pages 52-68. Springer, 2005.

12. V. Braberman, F. Fernández, D. Garbervetsky, and S. Yovine. Symbolic prediction of dynamic memory requirements. In ISMM 2008, 2008.

13. Victor Braberman, Diego Garbervetsky, and Sergio Yovine. A static analysis for synthesizing parametric specifications of dynamic memory consumption. Journal of Object Technology, 5(5):31-58, Jun 2006.

14. Philippe Clauss. Counting solutions to linear and nonlinear constraints through Ehrhart polynomials: applications to analyze and transform scientific programs. In ICS '96: Proceedings of the 10th International Conference on Supercomputing, pages 278-285, 1996.

15. Philippe Clauss and Vincent Loechner. Parametric analysis of polyhedral iteration spaces. Journal of VLSI Signal Processing, 19:179-194, 1998.

16. Patrick Cousot and Nicolas Halbwachs. Automatic discovery of linear restraints among variables of a program. In POPL '78: Proceedings of the 5th Annual ACM Symposium on Principles of Programming Languages, pages 84-97. ACM Press, 1978 .

17. Eugène Ehrhart. Sur un problème de géométrie diophantienne linéaire. I. Polyèdres et réseaux. J. Reine Angew. Math., 226:1-29, 1967.

18. Eugène Ehrhart. Sur un problème de géométrie diophantienne linéaire. II. Systèmes diophantiens linéaires. J. Reine Angew. Math., 227:25-49, 1967.

19. Sumit Gulwani, Krishna K. Mehra, and Trishul M. Chilimbi. SPEED: precise and efficient static estimation of program computational complexity. In $P O P L$ '09: Proceedings of the 36th ACM SIGPLAN-SIGACT Symposium on Principles of Programming Languages, pages 127-139, 2009.

20. Jan Hoffmann and Martin Hofmann. Amortized resource analysis with polynomial potential. In ESOP 2010: Proceedings of the 19th European Symposium on Programming Languages and Systems, volume 6012 of Lecture Notes in Computer Science, pages 287-306. Springer, 2010.

21. Martin Hofmann and Steffen Jost. Static prediction of heap space usage for firstorder functional programs. In POPL '03: Proceedings of the 30th ACM SIGPLANSIGACT Symposium on Principles of Programming Languages, pages 185-197, 2003.

22. Samin Ishtiaq and Peter W. O'Hearn. BI as an assertion language for mutable data structures. In POPL '01: Proceedings of the 28th ACM SIGPLAN-SIGACT Symposium on Principles of Programming Languages, pages 14-26, January 2001. 
23. Dieter Kratsch, Ross M. McConnell, Kurt Mehlhorn, and Jeremy P. Spinrad. Certifying algorithms for recognizing interval graphs and permutation graphs. In SODA '03: Proceedings of the Fourteenth Annual ACM-SIAM Symposium on Discrete Algorithms, pages 158-167, 2003.

24. Christian Lengauer. Loop parallelization in the polytope model. In CONCUR '93, volume 715 of Lecture Notes in Computer Science, pages 398-416. Springer, 1993.

25. Vincent Loechner and Doran K. Wilde. Parameterized polyhedra and their vertices. Int. J. of Parallel Programming, 25:25-6, 1997.

26. Jesús A. De Loera, Raymond Hemmecke, Jeremiah Tauzer, and Ruriko Yoshida. Effective lattice point counting in rational convex polytopes. Journal of Symbolic Computation, 38:1273-1302, 2004.

27. Paul Lokuciejewski, Daniel Cordes, Heiko Falk, and Peter Marwedel. A fast and precise static loop analysis based on abstract interpretation, program slicing and polytope models. In CGO '09: Proceedings of the 2009 International Symposium on Code Generation and Optimization, pages 136-146, 2009.

28. Tyrrell B. McAllister. Coefficient functions of the Ehrhart quasi-polynomials of rational polygons. In ITSL, pages 114-118. CSREA Press, 2008.

29. Kurt Mehlhorn, Arno Eigenwillig, Kanela Kanegossi, Dieter Kratsch, Ross McConnel, Uli Meyer, and Jeremy Spinrad. Certifying algorithms (a paper under construction), 2005. http://www.mpi-inf.mpg.de/ ${ }^{\sim m e h l h o r n / f t p / ~}$ CertifyingAlgorithms.pdf.

30. Benoît Meister. Approximations of polytope enumerators using linear expansions. Technical report, Universite Louis Pasteur, May 2007.

31. George C. Necula. Proof-carrying code. In POPL '97: Proceedings of the 24th ACM SIGPLAN-SIGACT Symposium on Principles of Programming Languages, pages 106-119, 1997.

32. Louis-Noël Pouchet, Cédric Bastoul, Albert Cohen, and John Cavazos. Iterative optimization in the polyhedral model: part II, multidimensional time. SIGPLAN Not., 43(6):90-100, 2008.

33. William Pugh. Counting solutions to Presburger formulas: how and why. In PLDI '94: Proceedings of the ACM SIGPLAN 1994 Conference on Programming Language Design and Implementation, pages 121-134. ACM, 1994.

34. John C. Reynolds. Separation logic: A logic for shared mutable data structures. In Proceedings of 17th Annual IEEE Symposium on Logic in Computer Science, 2002.

35. Donald Sannella, Martin Hofmann, David Aspinall, Stephen Gilmore, Ian Stark, Lennart Beringer, Hans-Wolfgang Loidl, Kenneth MacKenzie, Alberto Momigliano, and Olha Shkaravska. Mobile resource guarantees. In Trends in Functional Programming, volume 6, pages 211-226. Intellect, 2007.

36. Robert Endre Tarjan. Amortized computational complexity. SIAM Journal on Algebraic and Discrete Methods, 6(2):306-318, 1985.

37. Sven Verdoolaege and Maurice Bruynooghe. Algorithms for weighted counting over parametric polytopes: A survey and a practical comparison. In The 2008 International Conference on Information Theory and Statistical Learning, pages 60-66, 2008.

38. Sven Verdoolaege, Rachid Seghir, Kristof Beyls, Vincent Loechner, and Maurice Bruynooghe. Analytical computation of Ehrhart polynomials: Enabling more compiler analyses and optimizations. In Proceedings of the 2004 International Conference on Compilers, Architecture, and Synthesis for Embedded Systems (CASES), pages 248-258, September 2004. 


\section{A Appendix: examples of polyhedral analysis}

\section{A.1 Gaussian elimination}

The code below is an implementation of Gaussian elimination for the solution of simultaneous linear equations. This is based on code which was downloaded from the WWW ${ }^{10}$, but it has been modified by adding println methods to give the analysis something to count, and by replacing references to A. length by an integer $\mathrm{N}$ since our analysis currently only takes account of program variables, and cannot deal with fields.

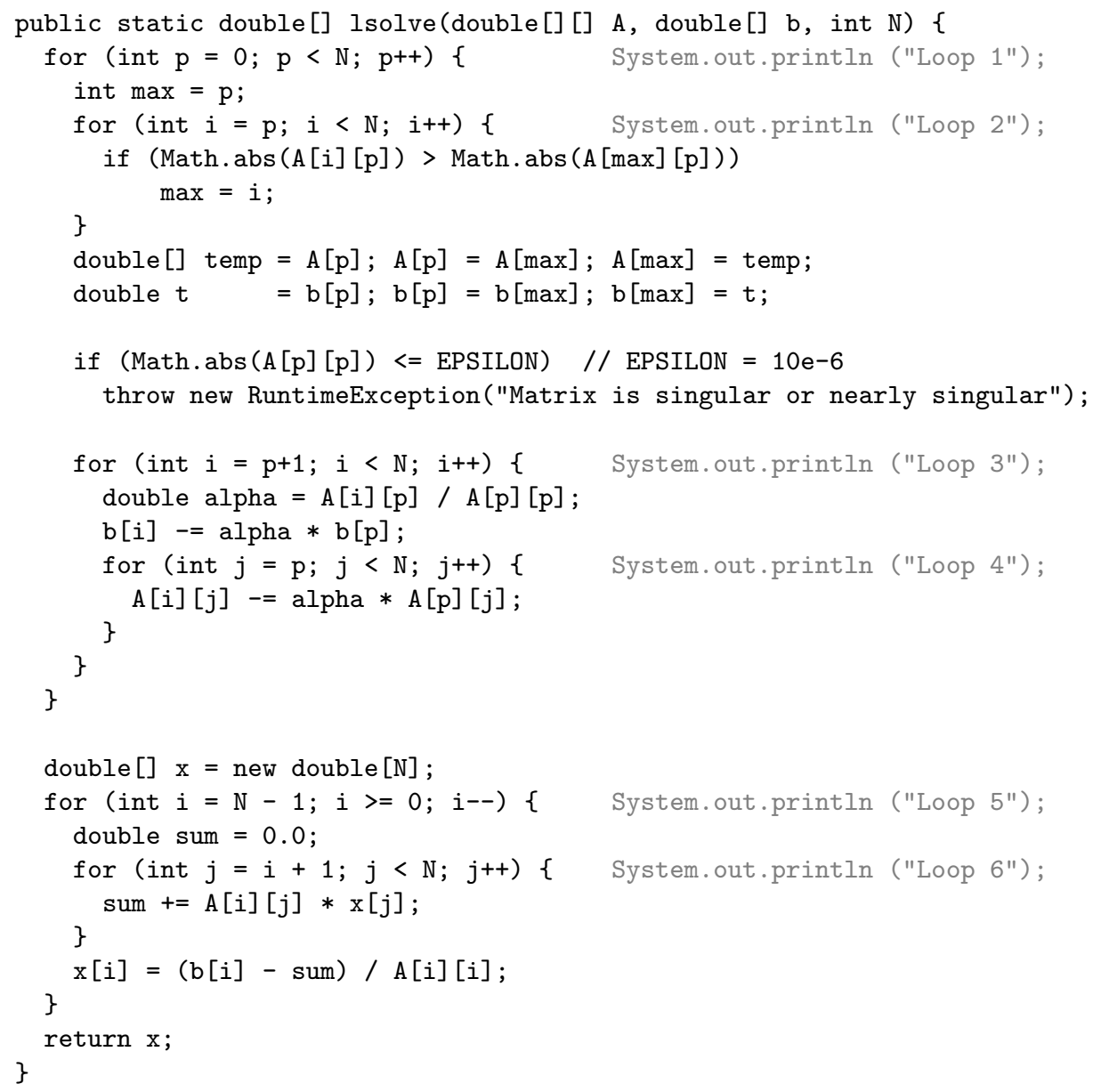

The output from the analysis appears below, with bounds on the number of calls to each println statement in the same order as in the program text. The analysis successfully finds tight bounds for the various nested loops.

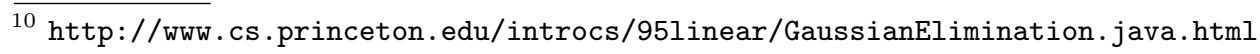




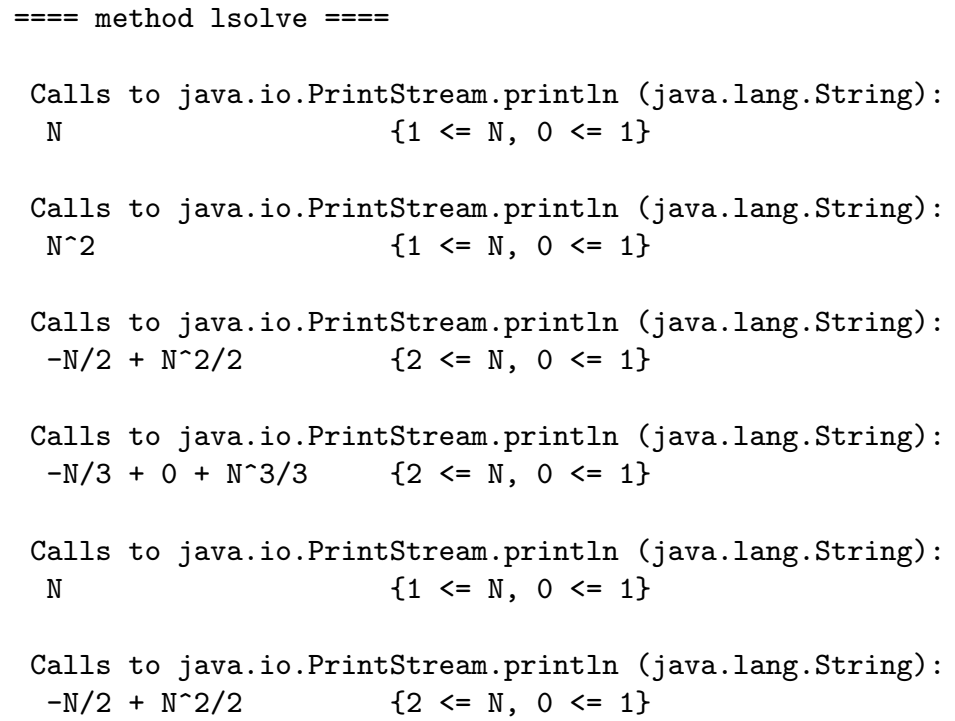

\section{A.2 Multiple parameters}

We also include a simple example involving multiple parameters which demonstrates the strength of the mathematical techniques underlying our analysis.

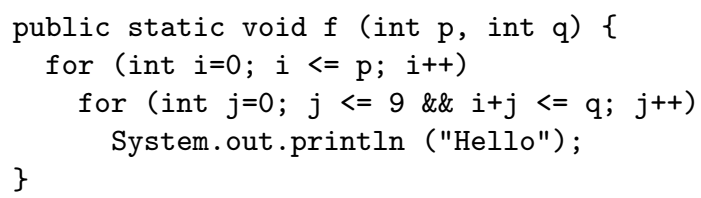

The number of iterations depends on the relative values of the arguments $p$ and q, with different Ehrhart polynomials applying for different combinations of arguments. The barvinok library is able to calculate these automatically, and comparatively little programming effort was required on our part to enable the analysis to find results of this type.

Calls to java.io.PrintStream.println (java.lang.String):

5 domains in $\mathrm{R}^{\wedge} 2$

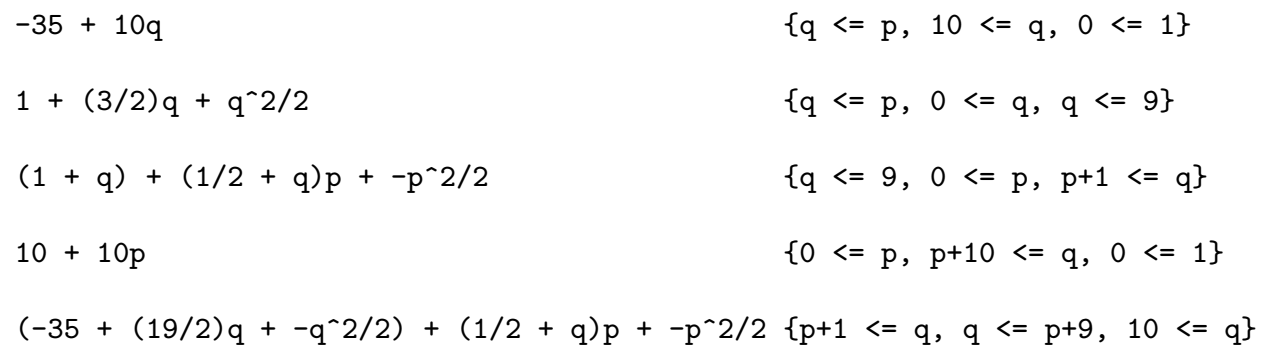

